\title{
A Novel Aerial Manipulation Design, Modelling and Control for Geometric CoM Compensation
}

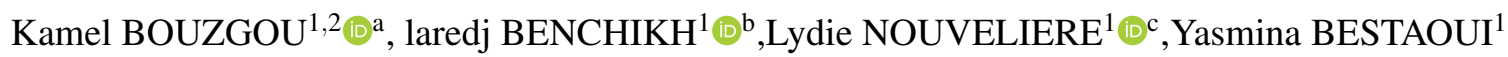 \\ (D) ${ }^{\mathrm{d}}$ and Zoubir AHMED-FOITIH ${ }^{2}$ (D) \\ ${ }^{1}$ IBISC, Univ Evry, Université Paris-Saclay, 91025, Evry, France. \\ ${ }^{2}$ LEPESA Laboratory, Faculty of Electrical Engineering,Department of electronics, USTO-MB,31000 Oran, Algerie. \\ kamel.bouzgou,laredj.benchikh,lydie.nouveliere@univ-evry.fr, zoubir.foitih@univ-usto.dz.
}

Keywords: UAVs, Aerial Robot, Flying manipulation, CLIKA, CoM compensation, Dynamic inverse control.

\begin{abstract}
This paper presents the design and modelling of a new Aerial manipulating system, that resolve a displacement of centre of gravity of the whole system with a mechanical device. A prismatic joint between the multirotor and a robotic arm is introduced to make a centre of mass as close as to the geometric centre of the whole system. This paper details also the geometric and dynamic modelling of a coupled system with a Lagrange formalism and control law with a Closed Loop Inverse Kinematic Algorithm (CLIKA). This dynamic inverse control is validated in a Simulink environment showing the efficiency of our approach.
\end{abstract}

\section{INTRODUCTION}

Unmanned Aerial Vehicles (UAVs) becomes an important scientific field, Interesting applications would be filming scenes and snapshots, exploring a wide area, observing aspects for civil and military tasks, then recently in the road traffic. The beginning of the robotics was to help the industrialists to make complex tasks in a fast and precise way, the environment was the ground, when the flying machines appeared, the researchers discovered another field of exploitation, the interaction with the environment was difficult, It began with the use of cameras and remote sensors without having direct contact with the environment or the target. Researchers are on the use of different mechanisms to interact on targets, this is where they created the domain of flying manipulators. In the last few years, it has emerged a need for the interaction of that UAVs with the environment that is not easily accessible by humans, for this, the researchers have used for transporting, manipulation and grasping a payload, several tools are used: magnet, cables, grippers and manipulators, also a combination of all that, to ensure the target tasks.

\footnotetext{
a (iD) https://orcid.org/0000-0003-2374-2149

b (iD https://orcid.org/0000-0002-4617-399X

c (iD https://orcid.org/0000-0003-0027-7192

d(D) https://orcid.org/0000-0001-7716-5952

e (iD) https://orcid.org/0000-0003-3121-9964
}

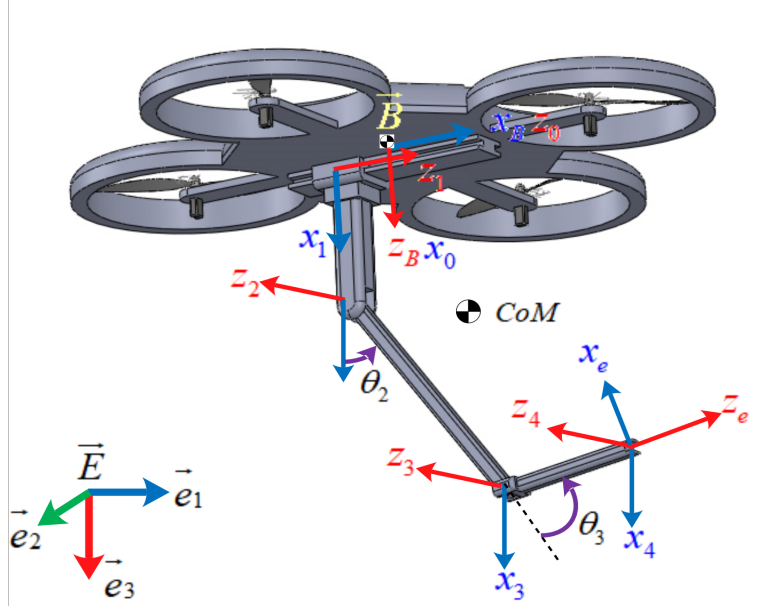

Figure 1: The structure of Q-PRR with principal frames

Almost systems in the literature consider a Quadrotor with manipulator arm and for $n$ DOF, a robot arm with revolute joints, And they place the system support which they can't generate large angles for the first joint, and the result will be a reduced workspace with a joint limit. Therefore it will be found that from second joint to $n$, are the real joints to generate a real workspace of the robot arm where the UAV be in the stable position.

A several projects in the word that deals the aerial manipulators are founded, from conception to control,

as an example, the ARCAS project, (Aerial 
Robotics Cooperative Assembly System)for assembly and structure construction, with multi-link manipulators, the AIRobots, Innovative aerial service robots for remote inspections by contact and the AIROARMS aerial robotic system with multiple arms for inspection and maintenance.

Firstly, the general related works are presented in Section 2, the design and modelling of the Q-PRR system is presented in Section 3, in Section 4 the inverse dynamic control with the closed loop inverse kinematics algorithm is presented, finally a conclusion is presented in the Section $\mathbf{5}$.

\section{RELATED WORKS}

In order to classify the different aerial manipulator, three main classes can be made for describe a flying manipulator according to the attached tools. UAV transporting payload with a flexible cable, in this case (Dai et al., 2014) can be cited The cable is modelled as a serial connection of arbitrary number of links.

Grasping, the multirotor is equipped with magnet to grasp object, or with a simple gripper(Escareno et al., 2014), also in (Srikanth et al., 2011) the author used a non prehensile manipulation with a single Dof to push an object in the desired direction, in (Yeol et al., 2017) author used a novel mechanical design with a single DOF for tentacle system for grasping objects, that structure system is cable-driven. UAV with a manipulator robot arm from 2..n-DOF, we can subdivide that in the two sub-classes.

Non-redundant robot arms with a degree of freedom $n<6$, the most papers are deals that structure, for 2DOF in (Aydemir et al., 2015, Kim et al., 2013), and in (Mello et al., 2015) authors used a 3-DOF robot arm with revolute joints, for 4-DOF in (JimenezCano et al., 2017) authors used a robot arm placed at the upper part of the UAV for bridge inspection. In (Kondak et al., 2013) they used a 5-DOF manipulator for interacting with environment. Redundant robot arm $n>6$, in (Huber et al., 2013) aerial manipulation with a 7-DOF industrial manipulator based on a main-tail-rotor helicopter, in (Danko and Oh, 2014)the Hyper-redundant manipulator with 9-DOF gives a large reachable spaces.

UAV with a different structure for grasping, transporting, manipulation an object, a delta structure fixed on side of UAV (Fumagalli et al., 2014), and parallel robot in (Cho and Shim, 2017; Danko et al., 2015), and interaction with object applying a forces and torque, a dual 4-dof arm on UAV in (Korpela et al., 2013).
The modified inertial parameters of the UAV to maintain the global system in a stable situation, to control it without having an important disturbance on the UAV base, for that, researchers developed a structure with moving UAV battery in one direction to maintain the CoG of that in a position as close as possible to the vertical axis that through centre gravity of overall system( Ruggiero et al., 2015), in (Kondak et al., 2013) authors used helicopter equipped with robot arm, the movement of the manipulator $\mathrm{CoG}$ while compensating the displacement of helicopter. The drawback of such structure, it is that a robot arm must be mounted on the specific UAV designed just for manipulation task, where the battery movement is very limited when the end-effector tried to reach a desired position and battery position can't ensure the alignment of CoM of UAV and robot arm.

In this a new structure of robot arm with a 3-DOF is developed, structure that can be fixed on any UAV, where the alignment of CoG of whole system can be ensured with a simple movement of robot arm and with one joint along one axis. This structure can be mounted on any UAV such as Quadrotor and helicopter or other heaving system, it offers several features such as, it works independently of UAV, whatever the UAV structure.

Ensure a wide workspace and a good stability of UAV in flying. Offers a large possibles configuration of robot arm, where a better for specified task and for desired position and orientation can be chosen.

Can consider that for given time and attitude, a base fixed robot arm with disturbance due to UAV oscillation, and compensates that for a small revolute joint displacement of second and third joints. The system can be stabilized by controlling a robot arm for a CoGs combinations, in (Lipiello and Ruggiero, 2012) authors are stabilized UAV CoG and robot arm by controlling the battery position. This strategy must have a combined kinematic model and decrease a number of possible solutions for position and orientation.

Only few works are using a prismatic joint. In (Backus and Dollar, 2017) researchers designed a dual arm with a Prismatic-Revolute-Revolute joints (PRR). This architecture is considered as a gripper for the grasping of objects, and it is adapted for different shapes and volumes. In this case each 3DOF robot arm is considered as a finger of a whole gripper.

A light-weight prototype 3-arms manipulator is used in (Orsag et al., 2013) to build an efficient system considered as legs of multirotor during the landing and handling operations.

In (Mersha et al., 2014) the authors have described the using of simplified 1D planar dynamic model. This 
allowed to provide an easy overview of the system dynamic, the proposed controller and the moving of the manipulator in a 1D plan. This work describes the appearance of a prismatic joint in the mathematical model of the system.

\section{MODELING}

The structure of the proposed aerial manipulator is composed of two parts, the multirotor which has number of rotors that $n_{r} \geq 4$, and the manipulator arm attached in the bottom, their geometric centres are considered in the same $z$ axis $\left(\overrightarrow{b_{3}}\right)$ of the mobile frame $\vec{B}$. The manipulator is composed of three degree of freedom (3-DOF), PrismaticRevolute-Revolute joints, called (Q-PRR), the first joint is prismatic and its axis $\left(x_{1}\right)$ is parallel to the $x$-axis of the multirotor mobile frame $\vec{B}$, this joint is considered actuated and it moves along the same axis, and it is bounded on both directions by a value $r_{0}$, the distance between two axes $x_{b}$ and $x_{1}$ is denoted by $\left(d_{0}\right)$, the second and third joints are revolute, its rotation axes $\left(z_{2}\right)$ and $\left(z_{3}\right)$, will be parallel to the $(y)$ axis of frame $\vec{B}$, where the manipulator arm motions are considered in the plan composed by $(x, z)$-axes of the mobile frame $\vec{B}$ Figure 1

Consider a system composed by a Multirotor vehicle equipped with a $n-$ DOF robotic arm attached to the bottom, depicted in Fig 1 Let $\vec{E}:\left\{\overrightarrow{e_{1}}, \overrightarrow{e_{2}}, \overrightarrow{e_{3}}\right\}$ be the inertial reference frame, let $\vec{B}:\left\{\overrightarrow{b_{1}}, \overrightarrow{b_{2}}, \overrightarrow{b_{3}}\right\}$ be the mobile frame placed at the vehicle center of mass, and let $\vec{O}_{i}$ be the body frame of $i-t h$ link, where $i=1, \ldots, n$ denotes the link number All body-fixed coordinate frames are located at the center of mass of their corresponding rigid bodies, respectively.

The position of the frame $\vec{B}$ with respect to the inertial frame $\vec{E}$, is given by the $(3 \times 1)$ vector denoted by $p_{b}$, while its orientation denoted by $R_{b}$ is described by the sequence of rotations $X Y Z$ about axes of the fixed frame, it can be computed via premultiplication of three elementary rotation $R_{\phi}$ about $x, R_{\theta}$ about $y$ and $R_{\psi}$ about $z$, where $\varphi_{b}=\left[\begin{array}{lll}\phi & \theta & \psi\end{array}\right]^{T}$ is the vector of roll-pitch-yaw Euler angles, the orientation matrix $R_{b}$ is given by:

$$
R_{b}=\left[\begin{array}{ccc}
c_{\theta} c_{\psi} & s_{\phi} s_{\theta} c_{\psi}-c_{\phi} s_{\psi} & c_{\phi} s_{\theta} c_{\psi}+s_{\phi} s_{\psi} \\
c_{\theta} s_{\psi} & s_{\phi} s_{\theta} s_{\psi}+c_{\phi} s_{\psi} & c_{\phi} s_{\theta} s_{\psi}-s_{\phi} c_{\psi} \\
-s_{\theta} & s_{\phi} c_{\theta} & c_{\phi} c_{\theta}
\end{array}\right]
$$

where $s_{*}=\sin (*), c_{*}=\cos (*), R_{\psi}, R_{\theta}, R_{\phi}$ and $R_{b}$ are matrices defined in the special orthogonal group $S O(3)$, which has the following property:

$$
S O(3)=\left\{R \in \mathbb{R}^{3 \times 3} \mid R^{T} R=I, \operatorname{det}(R)=1\right\}
$$

Let $R_{e}^{b}$ be the orientation matrix of a frame attached to the end-effector, and $p_{e}^{b}=\left[\begin{array}{lll}x_{e b} & y_{e b} & z_{e b}\end{array}\right]^{T}$ presents the position vector of origin of such a frame with respect to $\vec{B}$, and the absolute position vector and orientation matrix of the end-effector with respect to $\vec{E}$, is given by the $p_{e}=\left[\begin{array}{lll}x_{e} & y_{e} & z_{e}\end{array}\right]^{T}$ and $R_{e}$, respectively, where the pair $\left(p_{b}, R_{b}\right) \in S E(3)$ denotes the vector position given by $p_{b}=\left[\begin{array}{lll}x_{b} & y_{b} & z_{b}\end{array}\right]^{T}$, and the orientation matrix of the multirotor with respect to the inertial frame $\vec{E}$, the coordinate frame assignment is depicted in Figure (2).

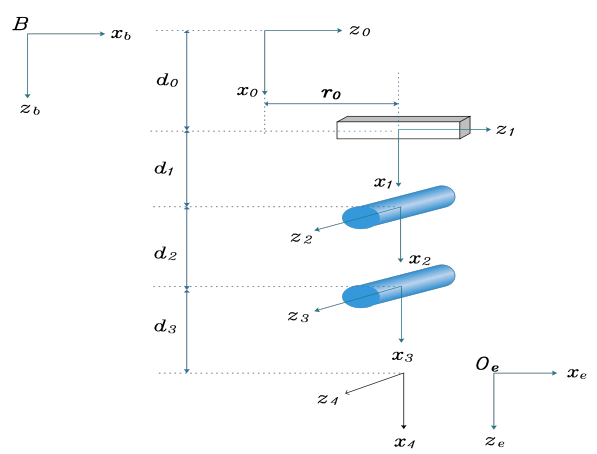

Figure 2: The coordinate frame assignment of system

\subsection{Kinematics}

The direct kinematic model (DK) consists of compute the position and attitude of end-effector named operational coordinates $\chi_{e}=\left[\begin{array}{cc}p_{e}^{T} & \varphi_{e}^{T}\end{array}\right]^{T}$, when $\chi_{e}$ is the $(6 \times 1)$ vector describing the system configuration of position and orientation with $\varphi_{e}$ is the endeffector orientation expressed via Euler angles (rollpitch-yaw).

As a function of the generalized joint variables, $\chi_{e}=$ $f(\xi): \mathbb{R}^{(6+n)} \longmapsto \mathbb{R}^{6}$, where $\xi$ is the $(6+n)$ vector of the generalized variables described as $\xi=$ $\left[\begin{array}{ll}q_{b}^{T} & q_{e b}^{T}\end{array}\right]^{T}$. Where $q_{b}, q_{e b}$ present the joint variables of the end-effector and multirotor frame with respect to $\vec{E}$ and $\vec{B}$, are expressed as $q_{b}=\left[\begin{array}{ll}p_{b}^{T} & \varphi_{b}^{T}\end{array}\right]^{T}$ and $q_{e b}=\left[\begin{array}{lll}q_{1} & \ldots & q_{n}\end{array}\right]^{T}$, respectively. Geometric methods are used by some researches, when the D-H method is used in this paper because of its recursive compute for modeling of any number of joints and links see (Siciliano et al., 2010). The DK is obtained by simple products of the homogeneous transformation matrices $A_{i}^{i-1}(i=1 \ldots n)$, from mobile frame $\vec{B}$ to the end-effector frame by:

$$
A_{e}=A_{b} A_{0}^{b} A_{1}^{0} A_{2}^{1} \ldots A_{n}^{n-1} A_{e}^{n}
$$

where $A_{0}^{b}$ is a constant homogeneous transformation describing the position and orientation of frame 0 
of robot arm base with respect to the mobile frame $\vec{B}$, those transformation matrices are obtained by using the Denavit-Hartenberg convention (Bouzgou and Ahmed-Foitih, 2014, Bouzgou et al., 2015) and methodology, and are listed in Table (1). The

Table 1: Denavit-Hartenberg (D-H) parameters of Q-PRR

\begin{tabular}{|c|c|c|c|c|c|}
\hline$i$ & $\sigma_{i}$ & $\alpha_{i}$ & $d_{i}$ & $\theta_{i}$ & $r_{i}$ \\
\hline 0 & 1 & 0 & $d_{0}$ & 0 & $r_{0}$ \\
\hline 1 & 0 & $\pi$ & $d_{1}$ & $\theta_{2}$ & 0 \\
\hline 2 & 0 & 0 & $d_{2}$ & $\theta_{3}$ & 0 \\
\hline 3 & 0 & 0 & $d_{3}$ & 0 & 0 \\
\hline
\end{tabular}

homogeneous matrix from the end-effector frame with respect to $\vec{B}$ frame is presented as:

$$
A_{e}^{b}=\left[\begin{array}{cccc}
c_{23} & 0 & s_{23} & r_{0}+d_{2} s_{2}+d_{3} s_{23} \\
0 & 1 & 0 & 0 \\
-s_{23} & 0 & c_{23} & d_{0}+d_{1}+d_{2} c_{2}+d_{3} c_{23} \\
0 & 0 & 0 & 1
\end{array}\right]
$$

Where $s_{23}=\sin \left(\theta_{2}+\theta_{3}\right)$ and $c_{23}=\cos \left(\theta_{2}+\theta_{3}\right)$

The position and orientation equations of end-effector expressed in $\vec{E}$ are written as follows:

$$
\begin{gathered}
p_{e}=p_{b}+R_{b} p_{e}^{b} \\
R_{e}=R_{b} \cdot R_{e}^{b}
\end{gathered}
$$

By denoting with $\dot{\varphi}_{b}$ the time derivative of $\varphi_{b}$.

$$
\omega_{b}=T\left(\phi_{b}\right) \dot{\varphi}_{b}
$$

Where $T\left(\phi_{b}\right)$ is the transformation matrix between the time derivative of the Euler angles $\varphi_{b}$ and the angular velocity of the multirotor $\omega_{b}$.

$$
T\left(\phi_{b}\right)=\left[\begin{array}{ccc}
1 & 0 & -s_{\theta} \\
0 & c_{\phi} & s_{\phi} c_{\theta} \\
0 & -s_{\phi} & c_{\phi} c_{\theta}
\end{array}\right]
$$

By differentiating (3), (4) and with taking into account (5), the translational and angular velocities of the end-effector with respect to $\vec{E}$ can be written as follows:

$$
\begin{gathered}
\dot{p}_{e}=\dot{p}_{b}-R_{b} \widehat{p}_{e b}^{b} T\left(\phi_{b}\right) \dot{\varphi}_{b}+R_{b} \dot{p}_{e b}^{b} \\
\omega_{e}=T\left(\phi_{b}\right) \dot{\varphi}_{b}+R_{b} \omega_{e b}^{b}
\end{gathered}
$$

Where $\dot{p}_{b}, \omega_{b}$ are the linear and angular velocities of the mobile frame $\vec{B}$ with respect to the $\vec{E}$ frame, respectively, and $\dot{p}_{e b}^{b}, \omega_{e b}^{b}$ are the translational and angular velocities of the end-effector with respect to the mobile frame $\vec{B}$. ( $): \mathbb{R}^{3} \longrightarrow S O(3)$, the hat map that transforms a vector in $\mathbb{R}^{3}$ to $(3 \times 3)$ Skew-symmetric matrix such that $\hat{x} y=x \times y, \forall x, y \in \mathbb{R}^{3}$ (Kamel et al., 2017).
Let be $v_{e b}^{b}=\left[\begin{array}{ll}\dot{p}_{e b}^{b T} & \omega_{e b}^{b T}\end{array}\right]^{T}$ the $(6 \times 1)$ vector of the generalized velocity of the end-effector with respect to $\vec{B}$, can be rewrite it in terms of $\dot{q}_{e b}$ via the jacobian matrix $\boldsymbol{J}_{e b}^{b}$ of the manipulator,

$$
v_{e b}^{b}=\boldsymbol{J}_{e b}^{b}\left(q_{e b}\right) \dot{q}_{e b}
$$

From 3 and 4 with the jacobian matrix equation 8 , the generalized velocity vector $v_{e}=\left[\begin{array}{ll}\dot{p}_{e}^{T} & \omega_{e}^{T}\end{array}\right]^{T}$, can be expressed as

$$
v_{e}=\boldsymbol{J}_{b} T_{A}\left(\phi_{b}\right) \dot{q}_{b}+\boldsymbol{J}_{e b} \dot{q}_{e b}
$$

Matrices $\boldsymbol{J}_{b}$ and $\boldsymbol{J}_{e b}$ are given by

$$
\begin{gathered}
\boldsymbol{J}_{b}=\left[\begin{array}{cc}
I_{3} & -R_{b} \widehat{p}_{e b}^{b} \\
O_{3} & I_{3}
\end{array}\right], \quad \boldsymbol{J}_{e b}=\left[\begin{array}{cc}
R_{b} & 0_{3} \\
O_{3} & R_{b}
\end{array}\right] \boldsymbol{J}_{e b}^{b} \\
\text { and } T_{A}\left(\phi_{b}\right)=\left[\begin{array}{cc}
I_{3} & 0_{3} \\
O_{3} & T\left(\phi_{b}\right)
\end{array}\right] \\
\boldsymbol{J}_{e b}=\left[\begin{array}{ccc}
1 & \left(d_{2} c 2+d_{3} c 23\right) & d_{3} c 23 \\
0 & 0 & 0 \\
0 & -\left(d_{2} s 2+d_{3} s 23\right) & -d_{3} s 23 \\
0 & 0 & 0 \\
0 & 1 & 1 \\
0 & 0 & 0
\end{array}\right]=\left[\begin{array}{l}
\boldsymbol{J}_{e b_{p}} \\
\boldsymbol{J}_{e b_{o}}
\end{array}\right]
\end{gathered}
$$

The existence of the inverse jacobian matrix is ensured when the end-effector avoids the set of singular positions in the $2 \mathrm{D}$ plane, is that by the resolution of $\operatorname{det}\left(\boldsymbol{J}_{e b_{p}}\right)=0$.

Therefore $J_{e b_{*}}^{-1}$ exists if $\theta_{2} \neq k \pi$, this is the singular position of the manipulator for the angles value $\theta_{2}$, where joints velocity in the operational space can not be ensured..

\subsection{1 inverse kinematics}

The inverse kinematics model consists the determination of joint variables required to achieve the given end-effector position and orientation, as a function, it can be written $\xi=f\left(p_{e}^{d}, R_{e}^{d}\right)$, where $p_{e}^{d}, R_{e}^{d}$ are the desired position and orientation of end-effector, respectively.

The position of the end-effector with respect to $\vec{B}$, $p_{e b} \in \mathbb{R}^{2}$, when the position of the multirotor with respect to the $\vec{E}$ is described by $\vec{B}$, with $p_{b} \in \mathbb{R}^{3}$, their workspaces is the disk for the robot arm, and a hemisphere for UAV.

Ruggiero Fabio and co-workers in (Ruggiero et al., 2015) are used a moving battery to counterweight the statics of the robotic arm. However, its movement can not be compensated if it moves will be faster than $15 \mathrm{~cm} / \mathrm{s}$, when the battery servo is limited to $\pi \mathrm{rad} / \mathrm{s}$, in addition the movement of the battery changes the CoG position of UAV. 
The structure of $Q-P R R$ is designed that the displacement of the arm does not change the CoG position of the multirotor, and can only displace the $\mathrm{CoG}$ of the system, in addition it can increase the linear velocity of the system in the $\overrightarrow{b_{2}}$, when the UAV and a slider joint are moved at the same time.

IK model computes the joint angles required to achieve the given position and orientation IK does not have a unique solution, the solutions which ensure collision avoidance, long battery life, minimum joint motion, and low torque values generate by robot arm applied on the multirotor, are considered more optimum.

The kinematic inverse system is obtained by using the pseudo-inverse of the matrix $\mathbf{J}$ presented in(Siciliano et al., 2010), then $\boldsymbol{J}^{\dagger}=\boldsymbol{J}^{T}\left(\boldsymbol{J} \boldsymbol{J}^{T}\right)^{-1}$.

The algorithm in (Arleo et al., 2013) is used, CLIKA: Closed loop inverse kinematics algorithm we obtain:

$$
\dot{\mathbf{q}}_{e}=\boldsymbol{J}^{\dagger}\left(\dot{x}_{e, d}-\boldsymbol{K} e\right)
$$

With $\boldsymbol{K}$ is a symmetric positive definite gain matrix. $e=x_{e}^{d}-x_{e}^{r}$, is the kinematic inversion error,we presented that in the Simulink block in Figure[3]

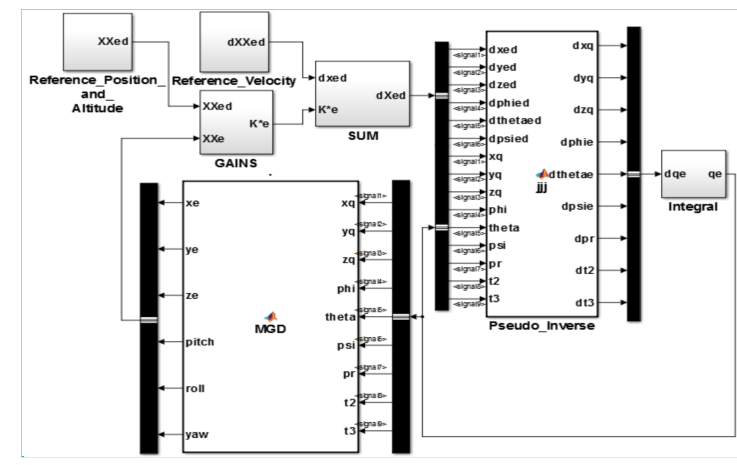

Figure 3: CLIKA Closed Loop Inverse Kinematic Algorithm

\subsection{Dynamics model}

The dynamic model of Q-PRR can be derived by considering the Lagrange formulation in details in (Lippiello and Ruggiero, 2012). The function of Lagrangian is then expressed by $\mathcal{L}=\mathcal{E}-\mathcal{U}$ where $\mathcal{E}$, $\mathcal{U}$ denote the kinematics and potential energy of the whole system, respectively. The Lagrange equations are given by

$$
\frac{d}{d t} \frac{\delta \mathcal{L}}{\delta \dot{\xi}_{i}}-\frac{\delta \mathcal{L}}{\delta \xi_{i}}=u_{i}
$$

Where $i=1, \ldots, 6+n$ is the $i-t h$ coordinate of $\xi$, and $u_{i}$ is the $((6+n) \times 1)$ vector of generalized forces and torques. The kinetic energy of the global system expressed in $\vec{B}$ frame is given by

$$
\mathcal{E}=\mathcal{E}_{b}+\sum_{i=1}^{n} \mathcal{E}_{c i}
$$

Where $\mathcal{E}_{b}$ is the kinetic energy of multirotor.

while $\mathcal{E}_{c i}$ is the kinetic energy of the $i$ link of manipulator arm.

The kinematic energy of multirotor can be expressed as

$$
\mathcal{E}_{b}=\frac{1}{2} m_{b} \dot{p}_{b}^{T} \dot{p}_{b}+\frac{1}{2} \omega_{b}^{T} R_{b} \boldsymbol{I}_{b} R_{b}^{T} \omega_{b}
$$

Where $\boldsymbol{I}_{b}$ and $m_{b}$ are the inertia matrix and the mass of the multirotor expressed with the respect to $\vec{B}$ frame, respectively. Taking into account that $Q=R_{b}^{T} T_{b}$, the kinetic energy of multirotor can be rewritten on the new form as

$$
\mathcal{E}_{b}=\frac{1}{2} m_{b} \dot{p}_{b}^{T} \dot{p}_{b}+\frac{1}{2} \dot{\varphi}_{b}^{T} Q^{T} \boldsymbol{I}_{b} Q \dot{\varphi}_{b}
$$

whereas, the kinetic energy of $i-t h$ link of the robotic manipulator is given by

$$
\mathcal{E}_{c i}=\frac{1}{2} m_{c i} \dot{p}_{c i}^{T} \dot{p}_{c i}+\frac{1}{2} \omega_{c i}^{T} R_{b} R_{c i}^{b} I_{c i}^{i}\left(R_{c i}^{b}\right)^{T} R_{b}^{T} \omega_{c i}
$$

from (12), 13) and (14) we obtain the kinetic energy of whole system and it can be written as,

$$
\begin{gathered}
\mathcal{E}=\frac{1}{2} \dot{\xi}^{T}\left(m_{Q} B_{Q 1}+\sum_{i=1}^{n} m_{c i} B_{c i 1} B_{c i 1}^{T}\right)+ \\
\frac{1}{2} \dot{\xi}^{T}\left(B_{Q 2} \boldsymbol{I}_{Q} B_{Q 2}^{T}+\sum_{i=1}^{n} B_{c i 2} \boldsymbol{I}_{c i}^{i} B_{c i 2}^{T}\right) \dot{\xi} \\
\mathcal{E}=\frac{1}{2} \dot{\xi}^{T} \boldsymbol{B} \dot{\xi}
\end{gathered}
$$

$\boldsymbol{B}$ is the $(3 \times 3)$ matrix of $(3 \times 3)$ inertia matrix elements, $\boldsymbol{B} \in \mathbb{M}^{(3 \times 3)}$ and $\boldsymbol{B}_{i j} \in \mathbb{R}^{(3 \times 3)}$. For more details see (Lippiello and Ruggiero, 2012). The potential energy of the whole system es given by the sum of that of the multirotor and that of each link of the manipulator arm.

$$
\mathcal{U}=\mathcal{U}_{b}+\sum_{i=1}^{n} \mathcal{U}_{c i}
$$

The potential energy of multirotor is given by

$$
\mathcal{P}_{b}=m_{b} g e_{3}^{T} p_{b}
$$

On the other hand, the potential energy of each link $i$ of manipulator is given by

$$
\mathcal{U}_{c i}=m_{c i} g e_{3}^{T}\left(p_{b}+R_{b} p_{c i}^{b}\right)
$$

The total potential energy of overall system can be get for a sum of (18) and (19) therefore:

$$
\mathcal{U}=m_{b} g e_{3}^{T} p_{b}+\sum_{i=1}^{n} m_{c i} g e_{3}^{T}\left(p_{b}+R_{b} p_{c i}^{b}\right)
$$


Where $g=9.8 \mathrm{~m} / \mathrm{s}^{2}$ is the gravity acceleration value and $e_{3}=\left[\begin{array}{lll}0 & 0 & 1\end{array}\right]$ unit vector along $z$ axis. Considering equations 12, 20 and 11, the dynamic model of the global system can be written as

$$
\boldsymbol{B}(\xi) \ddot{\xi}+\boldsymbol{C}(\xi, \dot{\xi}) \dot{\xi}+\boldsymbol{G}(\xi)=\boldsymbol{u}
$$

Where $\boldsymbol{G}$ is a $((6+n) \times 1)$ vector of gravitational terms given by deriving the potential energy as $\boldsymbol{G}(\xi)=\frac{\delta \mathcal{P}}{\delta \xi}$ And $\boldsymbol{C}$ is the matrix of Coriolis and centrifugal terms given by

$$
\boldsymbol{C}_{i j}=\sum_{k=1}^{6+n} \frac{1}{2}\left(\frac{\delta b_{i j}}{\delta \xi_{k}}+\frac{\delta b_{i k}}{\delta \xi_{j}}+\frac{\delta b_{j k}}{\delta \xi_{i}}\right) \dot{\xi}_{k}
$$

Where $b_{i} j$ is the generic element of $\boldsymbol{B}$, and $\boldsymbol{u}_{i}$ is the vector of generalized forces at the $i-t h$ joint level.

$$
\begin{gathered}
\boldsymbol{u}=\left[\begin{array}{c}
\boldsymbol{u}_{f_{b}} \\
\boldsymbol{u}_{\tau_{b}} \\
\boldsymbol{u}_{\mu}
\end{array}\right]=\left[\begin{array}{c}
R_{b} f_{b} \\
R_{b}^{T} T_{b} \tau_{b} \\
\mu
\end{array}\right]=\operatorname{diag}\left(R_{b}, Q, I_{n}\right) \\
f_{b}=\left[\begin{array}{c}
0 \\
0 \\
f_{b z}
\end{array}\right], \quad \tau_{b}=\left[\begin{array}{c}
\tau_{\phi} \\
\tau_{\theta} \\
\tau_{\psi}
\end{array}\right], \quad \mu=\left[\begin{array}{c}
f_{r_{0}} \\
\tau_{\theta_{2}} \\
\tau_{\theta_{3}}
\end{array}\right] \\
{\left[\begin{array}{c}
f_{b z} \\
\tau_{b}
\end{array}\right]=\left[\begin{array}{cccc}
1 & 1 & 1 & 1 \\
0 & l & 0 & -l \\
-l & 0 & l & 0 \\
c & -c & c & -c
\end{array}\right]\left[\begin{array}{l}
f_{1} \\
f_{2} \\
f_{3} \\
f_{4}
\end{array}\right]}
\end{gathered}
$$

Where $l$ is the distance from each motor to the multirotor centre of mass. When $c$ is the drag factor.

\section{CONTROLLER DESIGN}

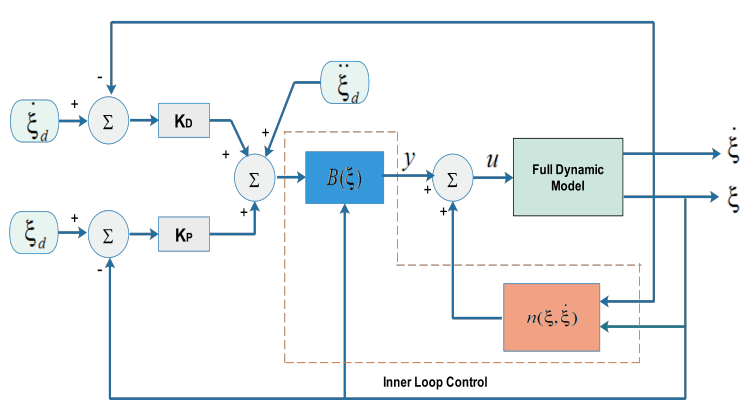

Figure 4: The control block diagram of Q-PRR

Dynamic inversion control is an approach founded to find a control vector $u$, as a function of the system state, where a feedback linearization loop is applied to the tracking outputs of the desired values, usage requires the selection of the output control variables. The possibility of finding such a linearizing controller is guaranteed by the particular form of system dynamics.
Which is able to realize an input/output relationship of linear type, in other words, we need to use an exact linearization of system dynamics obtained by means of a non-linear state feedback instead of an approximate linearization.

The possibility of finding such a linearizing controller is guaranteed by the particular form of system dynamics.

In fact, the equation in 22 is linear in the control $u$ and has a full-rank matrix $\boldsymbol{B}(\xi)$ which can be inverted for any manipulator configuration.

$$
\begin{gathered}
\boldsymbol{B}(\xi) \ddot{\xi}+\boldsymbol{n}(\xi,(\dot{\xi}))=\boldsymbol{u} \\
\boldsymbol{n}(\xi,(\dot{\xi}))=\boldsymbol{C}(\xi,(\dot{\xi}))(\dot{\xi})+\boldsymbol{g}(\xi)
\end{gathered}
$$

The control vector is chosen as follow:

$$
\begin{gathered}
\boldsymbol{u}=\boldsymbol{B}(\xi) \boldsymbol{y}+\boldsymbol{n}(\xi, \dot{\xi}) \\
\boldsymbol{y}=\ddot{\xi}_{d}+K_{P} \tilde{\xi}+K_{D} \dot{\tilde{\xi}}
\end{gathered}
$$

where $\boldsymbol{y}$ represents a new input vector $\tilde{\xi}=\xi_{d}-\xi$ and $\dot{\xi}=\dot{\xi}_{d}-\dot{\xi}$, And $K_{P}$ and $K_{D}$ are diagonal matrices with a positive gain. Two results to be compared for

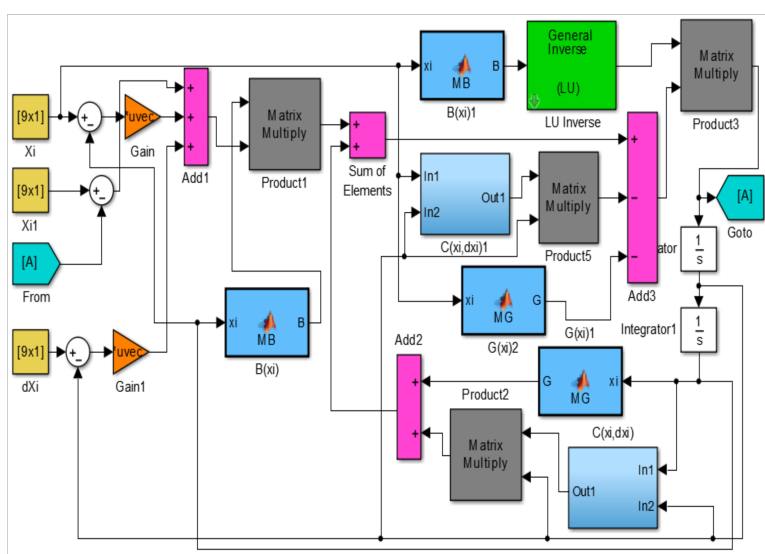

Figure 5: Block diagram of the proposed controller

a robot arm move to the desired position and in the only one configuration, the first case is when the multirotor is considered as in the stable situation, torques and forces are neglected, for a desired accuracy and position, the results are shown below in Figures. Two scenarios are realized.

The first Fig. 6, represents a static manipulator arm and its goal is to let the multirotor reaching a desired position. The second Fig. 7 represents a static multirotor and its goal is to let the manipulator arm reaching a desired position.

The robot design using SolidWorks and its all parameters is presented in the table 2 
Table 2: Dynamic parameters of Links

\begin{tabular}{cccccc}
\hline Link & mass & length & $I_{x x}$ & $I_{y y}$ & $I_{z z}$ \\
\hline \hline Base & 11.29 & 20 & 1324.16 & 1674.01 & 2561.03 \\
\hline 1 & 31.72 & 80 & 2283.37 & 19715.51 & 19807.8 \\
\hline 2 & 17.73 & 180 & 280.23 & 50131.61 & 50133.13 \\
\hline 3 & 9.61 & 95 & 206.92 & 7993.53 & 8059.21 \\
\hline
\end{tabular}

Gains of controller using Simulink are chosen in order to have not oscillations and to reach desired values as soon as possible.

$K_{D}=18 \boldsymbol{I}_{9}, K_{P}=30 \boldsymbol{I}_{9}$,

$x_{i}=[0 ; 0 ; 0 ; 0 ; 0 ; 0 ; 0.4 ; 0.2 ; 0.15]$,

$\dot{x}_{i}=[0 ; 0 ; 0 ; 0 ; 0 ; 0 ; 0 ; 0 ; 0]$,

$x_{i}=[2 ; 3 ; 5 ; 0 ; 0 ; 0 ; 0 ; 0 ; 0]$,

$\dot{x}_{i}=[0 ; 0 ; 0 ; 0 ; 0 ; 0 ; 0 ; 0 ; 0]$.
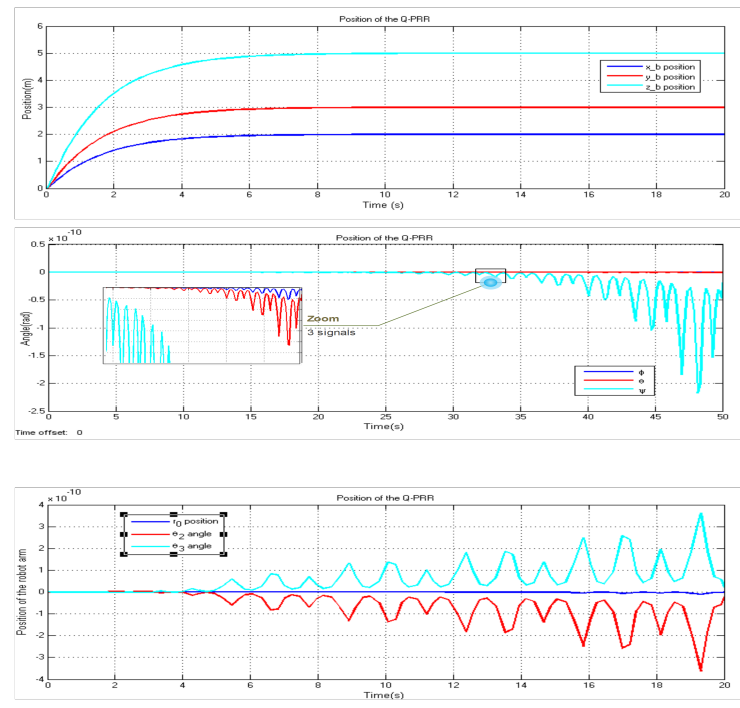

Figure 6: 1 st scenario

\section{CONCLUSIONS}

In this paper, a mathematical model for a whole system (multirotor and robot arm) is presented by using the Denavit-Hartenberg approach. The dynamic equation has been established according to the Lagrange's principle. The closed loop inverse kinematics algorithm is used and it was implemented in the control design. An inverse dynamic control is designed to achieve the desired performance for end effector target. Results show that torques and forces generated by the multirotor can disturb a manipula-
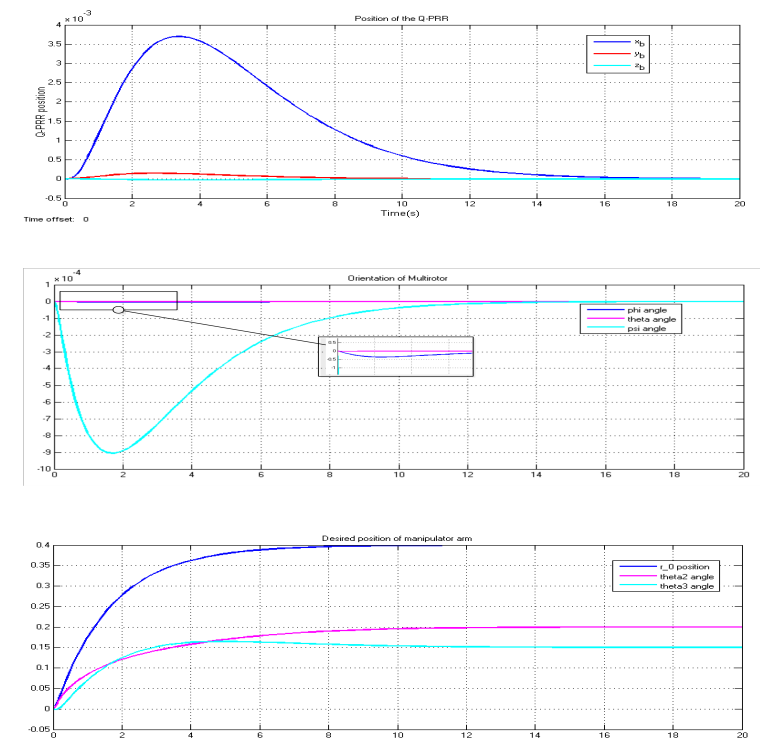

Figure 7: 2 nd scenario

tor motion, but the prismatic joint will work as an instantaneous corrector on the stability of the system without losing the correct configuration and without affecting the desired target. Our future works will present an another approach for a dynamic model by using a SimMechanics and VRML environment (Bouzgou et al., 2014).

\section{REFERENCES}

Arleo, G., Caccavale, F., Muscio, G., and Pierri, F. (2013). Control of quadrotor aerial vehicles equipped with a robotic arm. In Control \& Automation (MED), 2013 21st Mediterranean Conference on, pages 1174-1180. IEEE.

Aydemir, M., Arıkan, K. B., and İrfanoğlu, B. (2015). Disturbance rejection control of a quadrotor equipped with a 2 dof manipulator. In Machine Vision and Mechatronics in Practice, pages 91-103. Springer.

Backus, S. B. and Dollar, A. M. (2017). Design optimization of a prismatic-revolute-revolute joint hand for grasping from unconstrained vehicles. In ASME 2017 International Design Engineering Technical Conferences and Computers and Information in Engineering Conference, pages V05BT08A002-V05BT08A002. American Society of Mechanical Engineers.

Bouzgou, K. and Ahmed-Foitih, Z. (2014). Geometric modeling and singularity of 6 dof fanuc 200ic robot. In Innovative Computing Technology (INTECH), 2014 Fourth International Conference on, pages 208-214. IEEE.

Bouzgou, K., Ahmed-Foitih, Z., and Oran-Algeria, U. M. (2014). Singularity analysis and illustration of inverse kinematic solutions of 6 dof fanuc 200 ic robot in vir- 
tual environment. Journal of Intelligent Computing, 5(3):91-105.

Bouzgou, K., Amar, R. H. E., and Ahmed-Foitih, Z. (2015). Virtual reality simulation and singularity analysis of 3-rrr translational parallel robot. In Innovative Computing Technology (INTECH), 2015 Fifth International Conference on, pages 61-66. IEEE.

Cho, S. and Shim, D. H. (2017). Development of a vision-enabled aerial manipulator using a parallel robot. TRANSACTIONS OF THE JAPAN SOCIETY FOR AERONAUTICAL AND SPACE SCIENCES, AEROSPACE TECHNOLOGY JAPAN, 15(APISAT2016):a27-a36.

Dai, S., Lee, T., and Bernstein, D. S. (2014). Adaptive control of a quadrotor uav transporting a cable-suspended load with unknown mass. In Decision and Control (CDC), 2014 IEEE 53rd Annual Conference on, pages 6149-6154. IEEE.

Danko, T. W., Chaney, K. P., and Oh, P. Y. (2015). A parallel manipulator for mobile manipulating uavs. In Technologies for Practical Robot Applications (TePRA), 2015 IEEE International Conference on, pages 1-6. IEEE.

Danko, T. W. and Oh, P. Y. (2014). Design and control of a hyper-redundant manipulator for mobile manipulating unmanned aerial vehicles. Journal of Intelligent \& Robotic Systems, 73(1-4):709.

Escareno, J., Flores, G., Rakotondrabe, M., Romero, H., Lozano, R., and Rubio, E. (2014). Task-based control of a multirotor miniature aerial vehicle having an onboard manipulator. In Unmanned Aircraft Systems (ICUAS), 2014 International Conference on, pages 857-863. IEEE.

Fumagalli, M., Naldi, R., Macchelli, A., Forte, F., Keemink, A. Q., Stramigioli, S., Carloni, R., and Marconi, L. (2014). Developing an aerial manipulator prototype: Physical interaction with the environment. IEEE robotics \& automation magazine, 21(3):41-50.

Huber, F., Kondak, K., Krieger, K., Sommer, D., Schwarzbach, M., Laiacker, M., Kossyk, I., Parusel, S., Haddadin, S., and Albu-Schäffer, A. (2013). First analysis and experiments in aerial manipulation using fully actuated redundant robot arm. In Intelligent Robots and Systems (IROS), 2013 IEEE/RSJ International Conference on, pages 3452-3457. IEEE.

Jimenez-Cano, A., Heredia, G., and Ollero, A. (2017). Aerial manipulator with a compliant arm for bridge inspection. In Unmanned Aircraft Systems (ICUAS), 2017 International Conference on, pages 1217-1222. IEEE.

Kamel, B., Yasmina, B., Laredj, B., Benaoumeur, I., and Zoubir, A.-F. (2017). Dynamic modeling, simulation and pid controller of unmanned aerial vehicle uav. In Innovative Computing Technology (INTECH), 2017 Seventh International Conference on, pages 64-69. IEEE.

Kim, S., Choi, S., and Kim, H. J. (2013). Aerial manipulation using a quadrotor with a two dof robotic arm. In Intelligent Robots and Systems (IROS), 2013 IEEE/RSJ International Conference on, pages 49904995. IEEE.
Kondak, K., Krieger, K., Albu-Schaeffer, A., Schwarzbach, M., Laiacker, M., Maza, I., Rodriguez-Castano, A., and Ollero, A. (2013). Closed-loop behavior of an autonomous helicopter equipped with a robotic arm for aerial manipulation tasks. International Journal of Advanced Robotic Systems, 10(2):145.

Korpela, C., Orsag, M., Pekala, M., and Oh, P. (2013). Dynamic stability of a mobile manipulating unmanned aerial vehicle. In germany 2013, I., editor, Robotics and Automation (ICRA), 2013 IEEE International Conference on, pages 4922-4927. IEEE.

Lipiello, V. and Ruggiero, F. (2012). Exploiting redundancy in cartesian impedance control of uavs equipped with a robotic arm. In RSJ International Conference on Intelligent Robots and Systems, pages 3768-3773.

Lippiello, V. and Ruggiero, F. (2012). Cartesian impedance control of a uav with a robotic arm. IFAC Proceedings Volumes, 45(22):704-709.

Mello, L. S., Aldorno, B., and Raffo, G. V. (2015). Wholebody modeling and control of an unmanned aerial manipulator. In the XII Brazilian Symposium of Intelligent Automation (XII SBAI). Natal.

Mersha, A. Y., Stramigioli, S., and Carloni, R. (2014). Exploiting the dynamics of a robotic manipulator for control of uavs. In Robotics and Automation (ICRA), 2014 IEEE International Conference on, pages 17411746. IEEE.

Orsag, M., Korpela, C., and Oh, P. (2013). Modeling and control of mm-uav: Mobile manipulating unmanned aerial vehicle. Journal of Intelligent \& Robotic Systems, pages 1-14.

Ruggiero, F., Trujillo, M. A., Cano, R., Ascorbe, H., Viguria, A., Peréz, C., Lippiello, V., Ollero, A., and Siciliano, B. (2015). A multilayer control for multirotor uavs equipped with a servo robot arm. In Robotics and Automation (ICRA), 2015 IEEE International Conference on, pages 4014-4020. IEEE.

Siciliano, B., Sciavicco, L., Villani, L., and Oriolo, G. (2010). Robotics: modelling, planning and control. Springer Science \& Business Media.

Srikanth, M. B., Soto, A., Annaswamy, A., Lavretsky, E., and Slotine, J.-J. (2011). Controlled manipulation with multiple quadrotors. In AIAA Conf. on Guidance, Navigation and Control.

Yeol, J. W., Toohey, D., and Hwang, Y.-W. (2017). Design and analysis of a multiple tentacle system for mobile manipulation in micro aerial vehicles. Procedia Computer Science, 105:7-13. 